\title{
The Effect of Ultrasound-Guided Lung Recruitment Maneuvers On Atelectasis In Lung-Healthy Patients Undergoing Laparoscopic Gynecologic Surgery: a Randomized Controlled Trial
}

Yi Liu

Chengdu Second People's Hospital

Jingyu Wang

The First Affiliated Hospital of China Medical University

Yong Wan

Affiliated Hospital of North Sichuan Medical College

Yuan Geng

Affiliated Hospital of North Sichuan Medical College

Yiran Zhang

Affiliated Hospital of North Sichuan Medical College

Hang Su

Affiliated Hospital of North Sichuan Medical College

Yujiao Yang ( $\nabla$ yangyujiao@nsmc.edu.cn )

Affiliated Hospital of North Sichuan Medical College

\section{Research Article}

Keywords: lung ultrasound, atelectasis, recruitment manoeuvres, PEEP

Posted Date: December 27th, 2021

DOI: https://doi.org/10.21203/rs.3.rs-1085907/v1

License: (c) (i) This work is licensed under a Creative Commons Attribution 4.0 International License.

Read Full License 


\section{Abstract}

\section{Background}

Atelectasis is a major cause of hypoxemia during general anesthesia and postoperative pulmonary complications (PPCs).Some previous reported that the combined use of lung recruitment procedures (LRMs) and positive end-expiratory pressure (PEEP) in mechanical ventilation mode contributes to the avoidance of PPCs in patients after general anesthesia, while others suggest that the use of LRMs makes patients more susceptible to hemodynamic disturbances and lung injury, and is of limited potential to decrease the incidence of PPCs. From this perspective, controversy exists as to whether LRMs should be routinely applied to surgical patients. More importantly, corresponding clinical studies are also lacking. Therefore, this trial was conducted with the aim of solving the above problem.

\section{Methods}

In current clinical trial, patients undergoing laparoscopic gynecologic surgery with healthy lungs were randomized to the recruitment maneuvers group (RM group; $6 \mathrm{~cm} \mathrm{H} \mathrm{H}_{2} \mathrm{O}$ PEEP and RMs) and the control

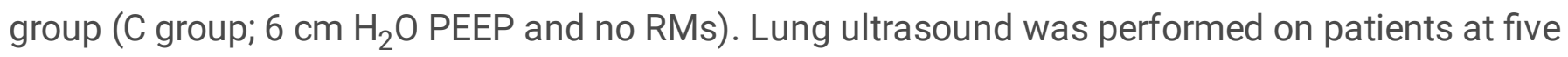
separate time points. During mechanical ventilation, patients in the RM group received ultrasound-guided pulmonary resuscitation when atelectasis was detected, while the $\mathrm{C}$ group did not intervene. Lung ultrasound scores were used to evaluate the incidence and severity of atelectasis.

\section{Results}

After LRMs, the incidence of atelectasis was significantly lower in the RM group (40\%) than in the C group (80\%) 15 minutes after arrival in the post-anesthesia care unit (PACU), and this difference did not persist for 24 hours after surgery. Meanwhile, postoperative pulmonary complications showed no difference between the two groups.

\section{Conclusions}

The combination of LRMs and PEEP decreased the incidence of atelectasis 15 minutes after admission to the PACU, but did not improve PPCs in adults with healthy lungs. Hence, for lung-healthy patients undergoing gynecological laparoscopic surgery, we do not recommend routine recruitment maneuvers. Trial registration: (prospectively registered): ChiCTR2000033529. Registered on 6/4/2020.

\section{Background}

Mechanical ventilation is a common respiratory support method in general anesthesia, and atelectasis is one of the common complications of mechanical ventilation that can be responsible for the development of PPCs. It has been shown that the incidence of atelectasis after general anesthesia is up to $90 \%$ [1] and that it can happen at the induction of anesthesia and last up to two days postoperatively, which prolongs hospitalization and increases medical costs [2]. Atelectasis is the main cause of hypoxemia during 
general anesthesia. Patients undergoing gynecological laparoscopic surgery usually need to establish carbon dioxide pneumoperitoneum and Trendelenburg position, which may further increase the incidence of atelectasis and the possibility of hypoxemia, thereby promoting PPCs $[3,4]$. The reason may be that carbon dioxide pneumoperitoneum and Trendelenburg position employed in laparoscopic gynecologic will cause the abdominal contents to push the diaphragm more cephalad, resulting in aggravated lung collapse, decreased functional residual capacity and more prone to atelectasis during perioperative period [5-7].

Although PEEP alone can improve intraoperative oxygenation [8], studies have shown that the combination of LRMs and PEEP can better improve oxygenation and reduce the incidence of PPCs in patients $[9,10]$. Recent studies suggest that the combination of LRMs and PEEP is helpful to prevent the occurrence of PPCs after general anesthesia in adults [11-13]. However, whether LRMs can reduce PPCs remains controversial [14-18]. Therefore, it is still unclear whether LRMs should be routinely applied in patients with normal lung function and whether it is beneficial for patients need to be further explored.

CT is the gold standard for clinical diagnosis of anencephaly, but its application is somewhat limited by its relative disadvantages such as high radiation, high transport risk, costly and timeconsuming examinations $[19,20]$. Lung ultrasound is a simple bedside imaging method that features the advantages of being affordable, radiation-free, and portable compared to CT. More importantly, pulmonary ultrasound can reliably diagnose and monitor atelectasis [21-23]. Therefore, the application of lung ultrasound may be more beneficial to patients in situations where mobility is limited, such as postoperative, catastrophic, or epidemic situations. In conclusion, our study aimed to assess the impact of LRMs by pulmonary ultrasound on the incidence of atelectasis in the included patients and to provide references for the application of LRMs to surgical patients.

\section{Methods}

\section{Study design}

The prospective randomized controlled study was conducted from June 2020 to October 2020 and approved by the Ethics Committee of the Affiliated Hospital of North Sichuan Medical College (Ethical No. 2020ER079-1). All subjects signed an informed consent form and completed registration with the China Clinical Trials Center (Approval No. ChiCTR2000033529). All methods were carried out in accordance with the Declaration of Helsinki.

\section{Study population}

Inclusion criteria were patients with healthy lungs aged 18 to 65 years, with a body mass index $(\mathrm{BMI})<35$ $\mathrm{kg} / \mathrm{m}^{2}$, American Society of Anesthesiologists (ASA) physical status I-II and undergoing gynecologic laparoscopic surgery. The pulmonary evaluation was performed by a specialized radiologist using $\mathrm{X}$-rays and CT on the included subjects. Exclusion criteria were that patients with pulmonary disease, cardiac disease, neuromuscular disease and corresponding surgical history, as well as respiratory tract infections 
were also excluded. Withdrawal criteria were as follows: (1) patients with preoperative ultrasound suggestive of pulmonary atelectasis; (2) surgical conversion from laparoscopic to open; (3) serious postoperative complications such as severe subcutaneous emphysema and pneumothorax.

\section{Randomisation and blinding}

According to the computerized randomization software (www.randomization.com), patients were randomly divided into $\mathrm{C}$ group and $\mathrm{RM}$ group in the ratio of 1:1. Group assignments were concealed in sealed envelopes that were opened after the anesthesiologist administered general anesthesia to the patient. Except for the anesthesiologist performing the induction of anesthesia and pulmonary ultrasound, who knows the grouping details, neither the patient nor the pulmonary ultrasound evaluator is aware of the details.

\section{Anesthesia and ventilation protocol}

All patients received standard general anesthetic protocol. Including $5 \mathrm{~L} \cdot \mathrm{min}^{-1}, 100 \%$ oxygen mask oxygen to nitrogen about $3 \mathrm{~min}$, induction of $0.04 \mathrm{mg} \cdot \mathrm{kg}^{-1}$ midazolam, $0.5 \mu \mathrm{g} \cdot \mathrm{kg}^{-1}$ sufentanil, $2 \mathrm{mg} \cdot \mathrm{kg}^{-1}$ propofol, $0.6 \mathrm{mg} \cdot \mathrm{kg}^{-1}$ rocuronium and the use of appropriate size of tracheal tube for intubation. The volumecontrolled mechanical ventilation mode was performed after intubation with a tidal volume of $8 \mathrm{ml} \cdot \mathrm{kg}^{-1}$, PEEP of $6 \mathrm{cmH}_{2} \mathrm{O}$ and 0.4 inspired oxygen fraction $\left(\mathrm{FIO}_{2}\right)$. The initial respiratory rate was set at 12 breaths $\min ^{-1}$ with an inspiratory: expiratory ratio of 1:2. The ventilator was adjusted to maintain an endtidal carbon dioxide pressure $\left(\mathrm{PETCO}_{2}\right)$ at $35-45 \mathrm{mmHg}$. Anesthesiologists can adjust $\mathrm{FIO}_{2}$ according to their experience when peripheral oxygen saturation $<90 \%$. Anesthesia was maintained by intravenous infusion of $0.1-0.3 \mu \mathrm{g} \cdot \mathrm{kg}^{-1} \cdot \mathrm{min}^{-1}$ remifentanil and $4-12 \mathrm{mg} \cdot \mathrm{kg}^{-1} \cdot \mathrm{h}^{-1}$ propofol, inhalation of $1 \%-3 \%$ sevoflurane. Bispectral index (BIS) was used to monitor the depth of anesthesia and maintain it at 40-60, timely supplement rocuronium to maintain adequate muscle relaxation. After spontaneous breathing recovery, neuromuscular blockade was reversed by neostigmine and glycopyrrolate. After extubation, the patient was sent to PACU, and oxygen was inhaled through the nose in PACU at a flow rate of $3 \mathrm{~L} \cdot \mathrm{min}^{-1}$. No analgesics were used postoperatively. Pulse oxygen saturation $\left(\mathrm{SpO}_{2}\right)$, mechanical ventilation time, PACU stay time and hospital stay time were recorded.

\section{Lung ultrasonography}

Lung ultrasound was performed by two trained and experienced anesthesiologists using an ultrasound machine (MINDRAY M9) with a probe of 2-5 MHz. Obtain sonograms at 5 scheduled time point: arrival in the operating suite (time point 1, T1), 1 minute after mechanical ventilation (time point 2, T2), at end of surgery (time point 3, T3), 15 min after arrival in the PACU (time point 4, T4), 24 hours after operation (time point $5, T 5$ ). Scanning was performed according to the lung ultrasound method described by Monastesse et al [23]. 
The thorax was divided into left and right lungs, a total of 12 quadrants. Left and right lungs were divided into upper and lower zones. Each side was divided into anterior, lateral and posterior zones by anterior and posterior axillary line. In the anterior and lateral regions, the probe is placed upright to the costal space, and in the posterior regions the probe is placed parallel to the costal space. In order to quantitatively evaluate the severity of atelectasis, we used the modified lung ultrasound score from Monastesse et al [23].

Furthermore, the degree of the lung ultrasound score (LUS) was divided into four grades and scored between 0 and 3: (0) 0-2 B lines; ( 1$) \geq 3$ B lines or 1 or multiple small subpleural consolidations separated by a normal pleural line; (2) multiple coalescent $B$ lines or multiple small subpleural consolidations separated by a thickened or irregular pleural line; and (3) consolidation or small subpleural consolidation of $>1 \times 2 \mathrm{~cm}$ in diameter. We defined atelectasis to be significant if any region had the lung ultrasound score $\geq 2$. The LUS was calculated by adding up the 12 individual quadrant scores, ranging from 0 to 36 points, with higher scores indicating chronic atelectasis.

\section{Study protocol}

When atelectasis appeared under ultrasound observation 1 minute after mechanical ventilation and at the end of surgery, patients of the RM group performed recruitment manoeuvres, placing the probe in the area of atelectasis, with airway pressure starting at $10 \mathrm{cmH}_{2} \mathrm{O}$ and increasing by $5 \mathrm{cmH}_{2} \mathrm{O}$ each time, up to $40 \mathrm{cmH}_{2} \mathrm{O}$, with a $0.4 \mathrm{FIO}_{2}$ was applied manually until no collapsed lung areas were visible on ultrasound. Then, the pressure was maintained for $40 \mathrm{~s}$. The mean arterial pressure and heart rate changed $<15 \%$.

\section{Perioperative observations}

The baseline characteristics were age, BMI, ASA classification. The observation indexes were LUS score and $\mathrm{SpO}_{2}$ at the above 5 time points, fluid volume, mechanical ventilation time, PACU residence time, hospitalization time and PPCs.

\section{Primary and secondary endpoints}

The primary endpoint was the incidence of intraoperative and postoperative pulmonary atelectasis and LUS score. The secondary endpoints were oxygen saturation, the incidence of intraoperative cardiovascular adverse reactions, PACU residence time, hospitalization time and PPCs.

\section{Sample size estimation}

We calculated the sample size using the data from previous studies. Yang et al.'s study shows that the frequency of atelectasis following a lung recruitment manoeuvre was $50 \%$, compared with $95 \%$ in adults after laparoscopic colorectal surgery who did not receive a recruitment manoeuvre [17]. The sensitivity of lung ultrasound detection of atelectasis was $88 \%$ [24]. According to our preliminary experiment, the incidence of atelectasis was $81 \%$ in lung-healthy patients after gynecologic laparoscopic surgery, which 
was reduced to $40 \%$ by LRMs. Therefore, if we assume an alpha error of 0.05 , power of $80 \%$ and allowing for a dropout rate of $10 \%$, the required sample size would be 20 patients per group.

\section{Statistical analysis}

Anthropometric data and demographics were collected from the individual patient. Following the normality of data testing, the Mann-Whitney $U$ test or $t$ test was used for intergroup comparisons as applicable. Friedman test or paired t test was used for intra-group comparison. Chi-square test or Fisher's exact test was used for categorical variables. A two-sided $P$ value less than 0.05 was considered significant unless Bonferroni adjustments were made. SPSS 25 and Graph Pad Prism 8 software were used for statistical analyses.

\section{Results}

From June to October 2020, 65 patients were included in this study. Among them, 23 patients were excluded for different reasons, as shown in Figure 1.

The remaining 42 patients were randomly divided into the RM group and the $\mathrm{C}$ group. One patient in the RM group withdrew from the trial due to severe postoperative subcutaneous emphysema resulting in poorly visualized lung ultrasound. Finally, 21 patients in the $\mathrm{C}$ group and 20 patients in the RM group were included in the analysis (Figure 1). A total of 2460 images were collected. Representative lung ultrasound images at various periods are shown in Figure 2.

Baseline characteristics of patients included in the study are shown in Table 1. There were no significant differences. Intraoperation parameters of the enrolled patients were shown in Table 2, which were also similar between the two groups.

Table 1

Baseline characteristics.

\begin{tabular}{|llll|}
\hline & C group, $\mathrm{n}=21$ & RM group, $\mathrm{n}=20$ & $\boldsymbol{P}^{*}$ \\
\hline Age $($ years $)$ & $38.14 \pm 10.85$ & $37.15 \pm 9.65$ & 0.759 \\
\hline BMI $\left(\mathrm{kg} / \mathrm{m}^{2}\right)$ & $21.66 \pm 3.07$ & $21.99 \pm 2.45$ & 0.057 \\
\hline ASA classification $(\mathrm{I} / \mathrm{II})$ & $0 / 21$ & $2 / 18$ & 0.142 \\
\hline
\end{tabular}

Date are presented as the mean \pm standard deviation unless otherwise stated. ${ }^{*}$, comparison between the two groups in per time point, with $\mathrm{P}<0.05$ considered significant. 
Table 2

Intraoperation parameters of the enrolled patients.

\begin{tabular}{|c|c|c|c|}
\hline & C group, $n=21$ & RM group, n=20 & $P *$ \\
\hline Total fluid intake (ml) & $1519.05 \pm 357.24$ & $1520.00 \pm 460.00$ & 0.574 \\
\hline Total fluid output (ml) & $332.62 \pm 198.86$ & $335.50 \pm 213.55$ & 0.974 \\
\hline $\mathrm{SpO}_{2}$ at $\mathrm{T} 1$ & $98.29 \pm 1.42$ & $98.60 \pm 0.82$ & 0.055 \\
\hline $\mathrm{SpO}_{2}$ at $\mathrm{T} 2$ & $99.76 \pm 0.44$ & $99.85 \pm 0.37$ & 0.163 \\
\hline $\mathrm{SpO}_{2}$ at T3 & $99.81 \pm 4.02$ & $99.85 \pm 3.66$ & 0.503 \\
\hline $\mathrm{SpO}_{2}$ at $\mathrm{T} 4$ & $97.90 \pm 1.55$ & $99.30 \pm 0.92$ & 0.148 \\
\hline $\mathrm{SpO}_{2}$ at $\mathrm{T} 5$ & $98.38 \pm 1.24$ & $99 \pm 0.80$ & 0.057 \\
\hline Mechanical ventilation duration (min) & $112.29 \pm 32.59$ & $123.05 \pm 41.41$ & 0.625 \\
\hline PACU stay duration (min) & $43.95 \pm 10.72$ & $41.55 \pm 9.24$ & 0.337 \\
\hline Hospital stay time (day) & $7.71 \pm 2.95$ & $7.00 \pm 3.61$ & 0.619 \\
\hline PPCs $( \pm)$ & $12 / 9$ & $11 / 9$ & 0.890 \\
\hline \multicolumn{4}{|c|}{$\begin{array}{l}\text { Date are presented as the mean } \pm \text { SD for continuous variables. PPCs, which included hypoxemia, } \\
\text { pulmonary infection, atelectasis, acute respiratory failure, ventilator-associated lung injury, and } \\
\text { neurogenic pulmonary edema. }{ }^{*} \text {, comparison between the two groups in per time point, with } P<0.05 \\
\text { considered significant. }\end{array}$} \\
\hline
\end{tabular}

As indicated in Table 3, the incidence of atelectasis in both groups of patients at each time point. At T2, there was no statistical difference in the incidence of atelectasis between the two groups, and this state persisted until after the first LRM. While after the second LRM, i.e., when observed at T4, the incidence of atelectasis in the RM group was lower than that in the $\mathrm{C}$ group『and this difference disappeared within 24 hours after operation (T5). 
Table 3

Incidence of atelectasis per group and per time point assessed by lung ultrasound.

\begin{tabular}{|llll|}
\hline & C group, $\mathbf{n = 2 1}$ & RM group, $\mathbf{n = 2 1}$ & $P^{*}$ \\
\hline T1n(\%) & $0(0)$ & $0(0)$ & \\
\hline T2n(\%) & $8(38)$ & $10(50)$ & 0.443 \\
\hline T3n(\%) & $16(76)$ & $19(95)$ & 0.089 \\
\hline T4n(\%) & $17(81)$ & $8(40)$ & 0.007 \\
\hline T5n(\%) & $12(57)$ & $11(55)$ & 0.890 \\
\hline $\begin{array}{l}\text { We defined atelectasis to be significant if any region had the lung ultrasound score } \geq 2 . \\
\text { between the two groups in per time point, with P<0.05 considered significant. }\end{array}$ \\
\hline
\end{tabular}

As illustrated in Figure 3, lung ultrasound score of 12 lung regions from T1 to T5 in both groups. Only at T3, lung ultrasound scores (LUSs) were lower in the RM group compared with the $\mathrm{C}$ group. The difference in LUSs between the two groups was similar to the difference in the incidence of atelectasis, which also disappeared 24 hours after operation. Besides, there was no difference in PPCs between the two groups as shown in table 2, and no side effects were found of recruitment manoeuvres in RM group.

Lung ultrasound score of anterior, lateral and posterior regions in 2 groups from T3 to T5, as shown in Figure 4a-c. LUSs of posterior regions were higher than those of the other two regions from T3 to T5 in the same group, with no significant difference between the anterior and lateral regions. In addition, there were difference of LUSs between the two groups at T4 was mainly due to the difference of lung ultrasound score in posterior regions.

\section{Discussion}

In this prospective randomized controlled trial, we found that the combination of ultrasound-guided recruitment manoeuvres and PEEP could reduce the incidence of atelectasis in patients with PACU compared with PEEP alone, but this difference disappeared 24h after operation.

The incidence of atelectasis in the PACU was as high as $81 \%$ in the $\mathrm{C}$ group, whereas the incidence of atelectasis in the PACU was reduced to $40 \%$ in the RM group by ultrasound-guided recruitment manoeuvres prior to extubation. Although the incidence of atelectasis was different in PACU, there was no difference in oxygen saturation and residence time between the two groups. The possible reasons are as follows. First, patients were treated with nasal catheter oxygen inhalation after operation, and atelectasis can be effectively alleviated. Therefore, patients in the RM group and the $\mathrm{C}$ group can have no clinical symptoms such as hypoxia. Second, patients with healthy lung have mild postoperative atelectasis, and the remaining healthy lung units can meet the compensation of the body, so there are no clinical symptoms. Interestingly, 24 hours post-operation, we found that the difference in the incidence of atelectasis and lung ultrasound scores between the two groups no longer existed. Moreover, we observed 
that the hospital stay time of patients was not different. Therefore, we believe that the combination of recruitment manoeuvres and PEEP was not more beneficial in preventing PPCs after gynecologic laparoscopic surgery in lung-healthy adults undergoing general anaesthesia.

Although it is reported that there are few side effects of recruitment manoeuvres $[25,26]$ and it is not found in this experiment, recruitment manoeuvres still has the risk of conducting hemodynamic disorder and ventilator-induced lung injury [25]. Therefore, we believe that ultrasound-guided recruitment maneuvers are not necessary for gynecologic laparoscopic surgery patients with normal lungs.

Some studies have shown that alveolar collapse and atelectasis can be formed within 5 min after general anesthesia induction and can persist postoperatively $[2,27]$. In present study, we found that in patients undergoing laparoscopic gynecological surgery, atelectasis can be formed after intubation and still not completely disappeared 24 hours postoperatively, which is in agreement with the results of the relevant studies $[2,27]$. is an important influencing factor for atelectasis, and related literature reports that the incidence of atelectasis during general anesthesia for laparoscopic surgery is positively correlated with $\mathrm{FIO}_{2}$ ranged from 0.4 to 1 . We administered pure oxygen during induction, which may be responsible for the development of alveolar collapse in patients several minutes after induction [28, 29].

Notably, there was no significant difference in the incidence of atelectasis between the two groups during operation. It can be seen that the combined application of ultrasound-guided lung recruitment maneuvers and PEEP after intubation had no significant effect on the progress of intraoperative atelectasis compared with the simple application of PEEP. Possible reasons for this are that intraoperative factors causing atelectasis continually take effect and that the short-term advantage from LRMs is not sustained, or because PEEP is relatively small and does not preserve alveolar opening. In our study, atelectasis mostly appears in the posterior regions of the lung (the patient's dorsum in a supine state), which is consistent with the view that atelectasis is mainly concentrated in the gravity-dependent area [23].

Before extubation, all sbujects in the RM group underwent the second LRM under ultrasound guidance until the atelectasis disappeared completely. When the lung ultrasound was reexamined in PACU after extubation, it was found that atelectasis was still present in $40 \%$ of the patients, and the alveolar collapsed again in a short time. The reason may be that the patients were in the PACU in the supine position with cranial displacement of the diaphragm, or other reasons such as postoperative extubation, obstruction by secretions, insufficient metabolism of anesthetic drugs, weak respiratory motility, and severe pain,etc [30].

Studies have shown that ultrasound-guided LRMs is more effective in diminishing atelectasis incidence in children than conventional methods [29], so we employed ultrasound-guided LRMs to minimize its undesirable effects. This study also suggests that it is feasible to monitor the changes of lung aeration in perioperative by lung ultrasound, and it can continuously and dynamically track the changes of aeration loss, which can be more widely applicable in the study of mechanical ventilation. 
Of course, this study has some limitations. 1. We administered pure oxygen from induction of anesthesia to the end of intubation in order to improve anesthetic safety, rather than optimal $\mathrm{FIO}_{2}$. The increase in $\mathrm{FIO}_{2}$ will contribute to the expansion of atelectasis area [31], possibly due to accelerated absorption of alveolar gas, resulting in absorptive atelectasis. 2. Our study included patients with healthy lungs and short surgery, and the incidence of PPCs was expected to be lower than after major surgery. Therefore, the preventive effect of ultrasound-guided LRMs on postoperative atelectasis may not be significant. It is necessary to further study the patients with high risk of PPCs.

\section{Conclusions}

In conclusion, our data indicate that the combination of LRMs and PEEP can reduce the incidence of atelectasis 15 min after arrival in PACU, but this advantage disappeared within 24 hours after operation, and it cannot improve PPCs in lung-healthy adults. Consequently, we do not recommend routine recruitment maneuvers for patients with healthy lungs undergoing gynecologic laparoscopic surgery.

\section{Abbreviations}

LRMs: lung recruitment maneuvers; PPCs: postoperative pulmonary complications; PEEP: positive end expiratory pressure; RM: recruitment maneuvers; PACU: post-anesthesia care unit; BMI: body mass index; ASA: American Society of Anesthesiologists; $\mathrm{FIO}_{2}$ : inspired oxygen fraction; $\mathrm{PETCO}_{2}$ : end-tidal carbon dioxide concentration; BIS: bispectral index; SpO2: pulse oxygen saturation; LUSs: lung ultrasound scores.

\section{Declarations}

\section{Ethics approval and consent to participate}

This study was approved by the ethics committee of the Affiliated Hospital of North Sichuan Medical College with approval number 2020ER079-1. The participants provided written consent. All methods were carried out in accordance with the Declaration of Helsinki.

\section{Consent for publication}

Not applicable.

\section{Availability of data and material}

The datasets used and/or analyzed during the current study are available from the corresponding author on reasonable request.

\section{Competing interests}

None. 


\section{Funding}

This research was funded by the Education \& teaching research and reform project of North Sichuan Medical College (21-31-048).

\section{Authors' contributions}

$Y L$ was responsible for the primary conception and design of the article. Initial drafts of the article were prepared by $Y$ L, Y W, J W, Y G, Y Z ang H S. Additions, modifications, and revisions critical for the important intellectual content of the article were performed by $Y Y$, including final approval of the version to be published. All authors read and approved the final manuscript.

\section{Acknowledgements}

None.

\section{References}

1. Gunnarsson L, Tokics L, Gustavsson H, Hedenstierna G. Influence of age on atelectasis formation and gas exchange impairment during general anaesthesia. $\mathrm{Br} \mathrm{J}$ Anaesth. [Journal Article; Research Support, Non-U.S. Gov't]. 1991 1991-04-01;66(4):423-32.

2. Wirth S, Biesemann A, Spaeth J, Schumann S. Pneumoperitoneum deteriorates intratidal respiratory system mechanics: an observational study in lung-healthy patients. SURG ENDOSC. [Journal Article; Observational Study]. 2017 2017-02-01;31(2):753-60.

3. Strang CM, Hachenberg T, Freden F, Hedenstierna G. Development of atelectasis and arterial to endtidal PCO2-difference in a porcine model of pneumoperitoneum. $\mathrm{Br} \mathrm{J}$ Anaesth. [Journal Article; Research Support, Non-U.S. Gov't]. 2009 2009-08-01;103(2):298-303.

4. Rubini A, Monte DD, Catena V. Effects of the pneumoperitoneum and Trendelenburg position on respiratory mechanics in the rats by the end-inflation occlusion method. ANN THORAC MED. [Journal Article]. 2012 2012-10-01;7(4):205-9.

5. Park JS, Ahn EJ, Ko DD, Kang H, Shin HY, Baek CH, et al. Effects of pneumoperitoneal pressure and position changes on respiratory mechanics during laparoscopic colectomy. Korean J Anesthesiol. [Journal Article]. 2012 2012-11-01;63(5):419-24.

6. Michelet P, D'Journo XB, Roch A, Doddoli C, Marin V, Papazian L, et al. Protective ventilation influences systemic inflammation after esophagectomy: a randomized controlled study. ANESTHESIOLOGY. [Journal Article; Randomized Controlled Trial; Research Support, Non-U.S. Gov't]. 2006 2006-11-01;105(5):911-9.

7. Wetterslev J, Hansen EG, Roikjaer O, Kanstrup IL, Heslet L. Optimizing peroperative compliance with PEEP during upper abdominal surgery: effects on perioperative oxygenation and complications in patients without preoperative cardiopulmonary dysfunction. Eur J Anaesthesiol. [Clinical Trial; Journal Article; Randomized Controlled Trial]. 2001 2001-06-01;18(6):358-65. 
8. Zhang L, Xiong W, Peng Y, Zhang W, Han R. The effect of an intraoperative, lung-protective ventilation strategy in neurosurgical patients undergoing craniotomy: study protocol for a randomized controlled trial. TRIALS. [Clinical Trial Protocol; Journal Article]. 2018 2018-02-02;19(1):85.

9. Tuncali B, Erol V, Zeyneloglu P. Effects of volume-controlled equal ratio ventilation with recruitment maneuver and positive end-expiratory pressure in laparoscopic sleeve gastrectomy: a prospective, randomized, controlled trial. TURK J MED SCI. [Comparative Study; Journal Article; Randomized Controlled Trial]. 2018 2018-08-16;48(4):768-76.

10. Li H, Zheng ZN, Zhang NR, Guo J, Wang K, Wang W, et al. Intra-operative open-lung ventilatory strategy reduces postoperative complications after laparoscopic colorectal cancer resection: A randomised controlled trial. Eur J Anaesthesiol. [Journal Article; Randomized Controlled Trial; Research Support, Non-U.S. Gov't]. 2021 2021-10-01;38(10):1042-51.

11. Hartland BL, Newell TJ, Damico N. Alveolar recruitment maneuvers under general anesthesia: a systematic review of the literature. Respir Care. [Journal Article; Review; Systematic Review]. 2015 2015-04-01;60(4):609-20.

12. Martin JB, Garbee D, Bonanno L. Effectiveness of positive end-expiratory pressure, decreased fraction of inspired oxygen and vital capacity recruitment maneuver in the prevention of pulmonary atelectasis in patients undergoing general anesthesia: a systematic review. JBI Database System Rev Implement Rep. [Journal Article; Review; Systematic Review]. 2015 2015-09-16;13(8):211-49.

13. Futier E, Constantin JM, Paugam-Burtz C, Pascal J, Eurin M, Neuschwander A, et al. A trial of intraoperative low-tidal-volume ventilation in abdominal surgery. N Engl J Med. [Journal Article; Multicenter Study; Randomized Controlled Trial]. 2013 2013-08-01;369(5):428-37.

14. Ferrando C, Soro M, Unzueta C, Suarez-Sipmann F, Canet J, Librero J, et al. Individualised perioperative open-lung approach versus standard protective ventilation in abdominal surgery (iPROVE): a randomised controlled trial. Lancet Respir Med. [Journal Article; Multicenter Study; Randomized Controlled Trial; Research Support, Non-U.S. Gov't]. 2018 2018-03-01;6(3):193-203.

15. Cui Y, Cao R, Li G, Gong T, Ou Y, Huang J. The effect of lung recruitment maneuvers on post-operative pulmonary complications for patients undergoing general anesthesia: A meta-analysis. PLOS ONE. [Journal Article; Meta-Analysis]. 2019 2019-01-20;14(5):e217405.

16. Yang Y, Geng Y, Zhang D, Wan Y, Wang R. Effect of Lung Recruitment Maneuvers on Reduction of Atelectasis Determined by Lung Ultrasound in Patients More Than 60 Years Old Undergoing Laparoscopic Surgery for Colorectal Carcinoma: A Prospective Study at a Single Center. Med Sci Monit. [Evaluation Study; Journal Article]. 2021 2021-01-18;27:e926748.

17. Genereux V, Chasse M, Girard F, Massicotte N, Chartrand-Lefebvre C, Girard M. Effects of positive endexpiratory pressure/recruitment manoeuvres compared with zero end-expiratory pressure on atelectasis during open gynaecological surgery as assessed by ultrasonography: a randomised controlled trial. Br J Anaesth. [Comparative Study; Journal Article; Randomized Controlled Trial; Research Support, Non-U.S. Gov't]. 2020 2020-01-01;124(1):101-9. 
18. Brismar B, Hedenstierna G, Lundquist H, Strandberg A, Svensson L, Tokics L. Pulmonary densities during anesthesia with muscular relaxation-a proposal of atelectasis. ANESTHESIOLOGY. [Journal Article; Research Support, Non-U.S. Gov't]. 1985 1985-04-01;62(4):422-8.

19. Schwebel C, Clec'H C, Magne S, Minet C, Garrouste-Orgeas M, Bonadona A, et al. Safety of intrahospital transport in ventilated critically ill patients: a multicenter cohort study*. CRIT CARE MED. [Journal Article; Multicenter Study; Research Support, Non-U.S. Gov't]. 2013 2013-0801;41(8):1919-28.

20. Yu X, Zhai Z, Zhao Y, Zhu Z, Tong J, Yan J, et al. Performance of Lung Ultrasound in Detecting PeriOperative Atelectasis after General Anesthesia. ULTRASOUND MED BIOL. [Journal Article; Observational Study; Research Support, Non-U.S. Gov't]. 2016 2016-12-01;42(12):2775-84.

21. Le Neindre A, Mongodi S, Philippart F, Bouhemad B. Thoracic ultrasound: Potential new tool for physiotherapists in respiratory management. A narrative review. J CRIT CARE. [Journal Article; Research Support, Non-U.S. Gov't; Review]. 2016 2016-02-01;31(1):101-9.

22. Monastesse A, Girard F, Massicotte N, Chartrand-Lefebvre C, Girard M. Lung Ultrasonography for the Assessment of Perioperative Atelectasis: A Pilot Feasibility Study. ANESTH ANALG. [Journal Article; Observational Study; Research Support, Non-U.S. Gov't]. 2017 2017-02-01;124(2):494-504.

23. Acosta CM, Maidana GA, Jacovitti D, Belaunzaran A, Cereceda S, Rae E, et al. Accuracy of transthoracic lung ultrasound for diagnosing anesthesia-induced atelectasis in children. ANESTHESIOLOGY. [Journal Article; Observational Study]. 2014 2014-06-01;120(6):1370-9.

24. Halbertsma FJ, van der Hoeven JG. Lung recruitment during mechanical positive pressure ventilation in the PICU: what can be learned from the literature? ANAESTHESIA. [Journal Article; Review]. 2005 2005-08-01;60(8):779-90.

25. Neves VC, Koliski A, Giraldi DJ. Alveolar recruitment maneuver in mechanic ventilation pediatric intensive care unit children. Rev Bras Ter Intensiva. [Journal Article]. 2009 2009-12-01;21(4):453-60.

26. Nimmagadda U, Salem MR, Crystal GJ. Preoxygenation: Physiologic Basis, Benefits, and Potential Risks. ANESTH ANALG. [Journal Article; Meta-Analysis; Review]. 2017 2017-02-01;124(2):507-17.

27. Kim BR, Lee S, Bae H, Lee M, Bahk JH, Yoon S. Lung ultrasound score to determine the effect of fraction inspired oxygen during alveolar recruitment on absorption atelectasis in laparoscopic surgery: a randomized controlled trial. BMC ANESTHESIOL. [Journal Article; Randomized Controlled Trial]. 2020 2020-07-18;20(1):173.

28. Wahba RW. Perioperative functional residual capacity. Can J Anaesth. [Journal Article; Review]. 1991 1991-04-01;38(3):384-400.

29. Lee JH, Choi S, Ji SH, Jang YE, Kim EH, Kim HS, et al. Effect of an ultrasound-guided lung recruitment manoeuvre on postoperative atelectasis in children: A randomised controlled trial. Eur $\mathrm{J}$ Anaesthesiol. [Journal Article; Randomized Controlled Trial]. 2020 2020-08-01;37(8):719-27.

30. Strandberg A, Tokics L, Brismar B, Lundquist H, Hedenstierna G. Atelectasis during anaesthesia and in the postoperative period. Acta Anaesthesiol Scand. [Comparative Study; Journal Article; Research Support, Non-U.S. Gov't]. 1986 1986-02-01;30(2):154-8. 
31. Hedenstierna G, Rothen HU. Atelectasis formation during anesthesia: causes and measures to prevent it. J CLIN MONIT COMPUT. [Journal Article; Review]. 2000 2000-07-06;16(5-6);329-335.

\section{Figures}

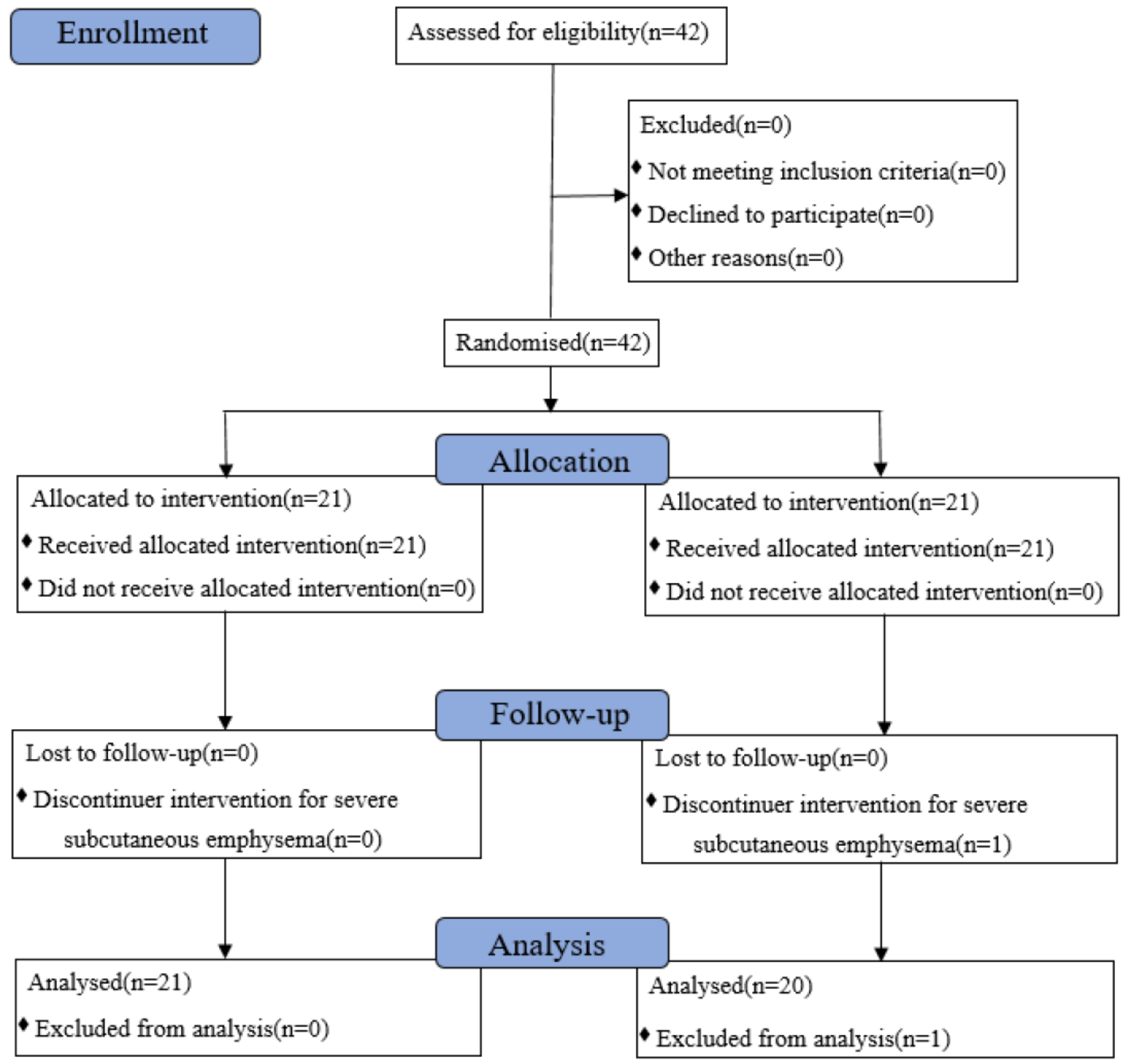

\section{Figure 1}

Flow diagram of patient screening and enrollment. 

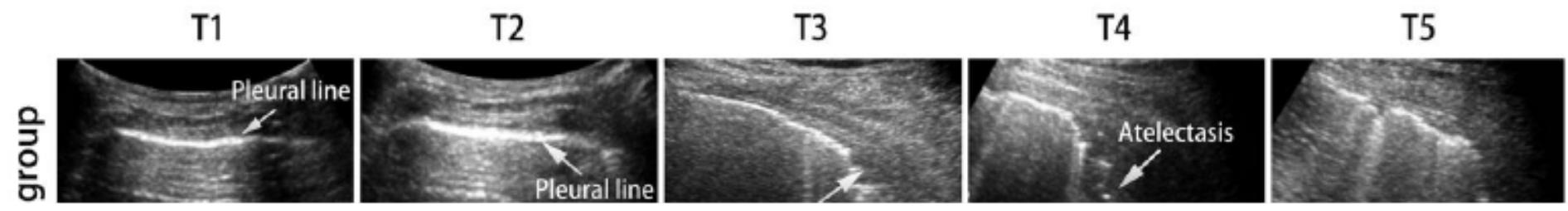

Figure 2

Lung ultrasound images of 1 representative patient per group.

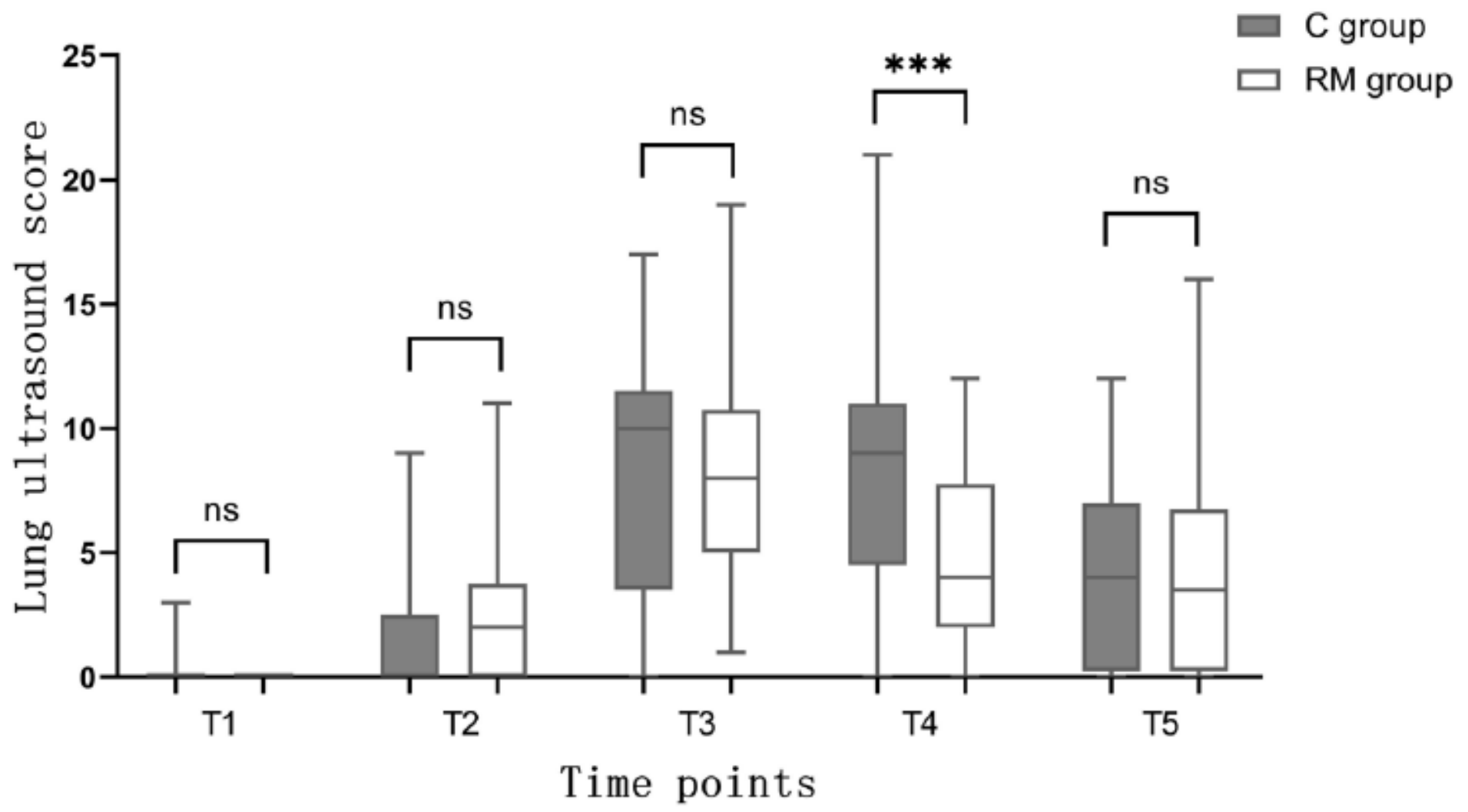

Figure 3

Lung ultrasound score of 12 lung regions from T1 to T5 in both groups. The box, whiskers and bold line across the box represent interquartile range, range and the median value, respectively. ns, no significance; $\star \star \star, P 囚 0.05$. 

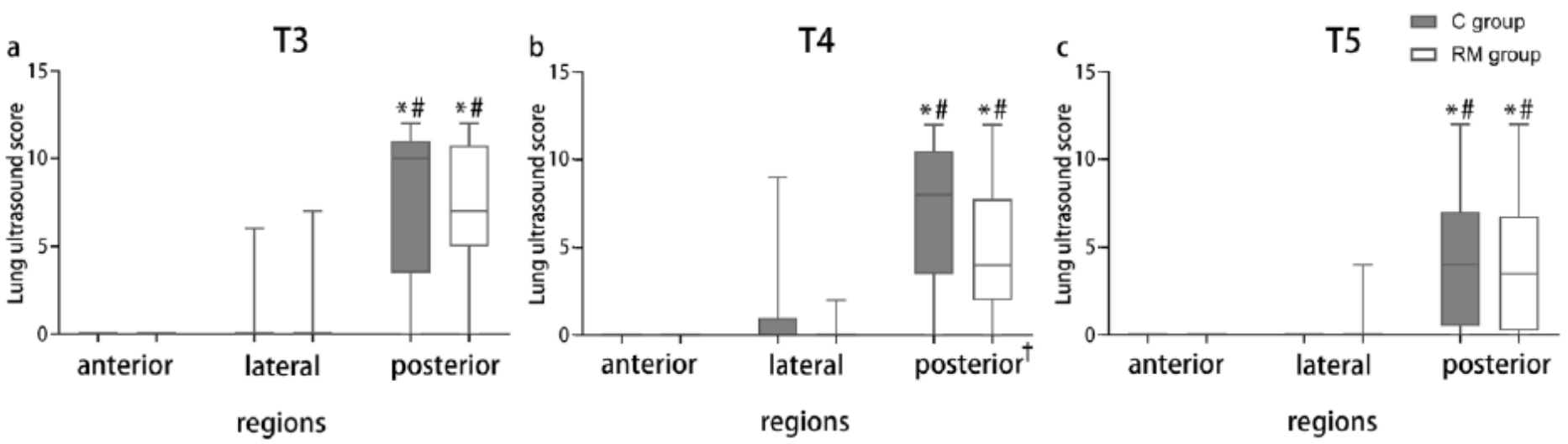

\section{Figure 4}

Lung ultrasound score of anterior, lateral and posterior regions in 2 groups from T3 to T5. The box, whiskers and bold line across the box represent interquartile range, range and the median value, respectively. ${ }^{*} \mathrm{P}<0.05$, posterior region vs. anterior region in the same group; ${ }^{\#} \mathrm{P}<0.05$, posterior region vs. lateral region in the same group; ${ }^{\dagger} \mathrm{P}<0.05$, RM group vs. $\mathrm{C}$ group. 